\title{
Suplementação Proteica não Aumenta a Concentração Plasmática de Óxido Nítrico em Homens Saudáveis
}

\author{
Protein Supplementation does not Increase Nitric Oxide Plasmatic \\ Concentration in Healthy Men
}

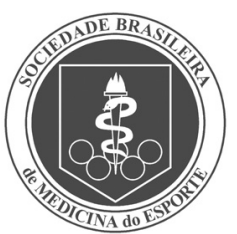

Artigo Original
Milton Rocha de Moraes ${ }^{1,2}$,

Marcelo Saldanha Aoki ${ }^{3}$,

Ronaldo de Carvalho Araújo ${ }^{2}$

Elisa Mieko Suemitsu Higa ${ }^{4}$,

Margaret Gori Mouro ${ }^{4}$,

Reury Frank Pereira Bacurau ${ }^{3}$

1. Laboratório de Aptidão Física, Faculdade de Educação Física, UMC, São Paulo, Brasil.

2. Departamento de Biofísica,

Unifesp, São Paulo, Brasil.

3. Curso de Ciências da Atividade

Física, Escola de Artes, Ciências e

Humanidades, USP, São Paulo, Brasil.

4. Departamento de Medicina

(Nefrologia), Unifesp, São Paulo,

Brasil.

Endereço para correspondência: Prof. Dr. Marcelo Saldanha Aoki.

Universidade de São Paulo.

Escola de Artes, Ciências e

Humanidades. Av. Arlindo Bettio,

1.000 .

Ermelino Matarazzo - 03828-000

- São Paulo. SP.

E-mail: saldanha.caf@usp.br

Submetido em 20/11/2006

Versão final recebida em 25/05/2008

Aceito em 09/08/2008

\begin{abstract}
RESUMO
Suplementos nutricionais, supostamente, capazes de potencializar a produção endógena de óxido nítrico (NO) têm experimentado crescente popularidade entre os indivíduos fisicamente ativos. Diante da carência de informações sobre o assunto, o objetivo do presente estudo foi avaliar o efeito de um suplemento comercial à base de proteínas e aminoácidos sobre a produção endógena de NO. Métodos: A amostra foi constituída de 12 homens sedentários, mas sem fatores de risco para doenças cardiovasculares. O protocolo de suplementação foi conduzido conforme o arranjo experimental duplo-cego cruzado. Os participantes receberam, aleatoriamente, placebo (PLA) ou suplemento proteico (SP), em dois momentos diferentes, separados por uma semana. Com o intuito de determinar a concentração plasmática de NO, amostras de sangue foram coletadas antes (24h e imediatamente antes) e depois (30 e 60 minutos) do consumo da substância PLA ou do SP. Resultados: Não foi observada alteração na concentração plasmática de NO após a ingestão do SP

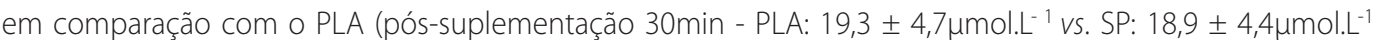
e pós-suplementação $60 \mathrm{~min}$ - PLA: $21,3 \pm 6,5 \mu \mathrm{mol}_{\text {.L }} \mathrm{L}^{-1}$ vs. SP: $20,3 \pm 4,9 \mu \mathrm{mol} . \mathrm{L}^{-1}$ ). Também não foi verificada alteração da pressão arterial. Conclusão: O suplemento nutricional à base de proteínas e aminoácidos, testado no presente estudo, não potencializou a produção endógena de NO.
\end{abstract}

Palavras-chave: aminoácidos, óxido nítrico, vasodilatação, pressão arterial.

\begin{abstract}
Nutritional supplements, theoretically able to increase endogenous nitric oxide (NO) production have experienced great popularity among physically active individuals. Aim: scientific evidence available regarding this issue is scarce. Therefore, the purpose of this study was to evaluate the effect of a dietary supplement commercialized as a nitric oxide booster. Materials and methods: twelve sedentary men with no risk factors for cardiovascular diseases were supplemented with placebo or protein in two different occasions. The present study was conducted in a cross double-blind design. In order to assess plasmatic NO concentration, blood samples were obtained before (24hs and immediately before) and after (30 and 60 minutes) consumption of placebo (PLA) or protein supplement (SP). Results: there was no difference in plasmatic nitric oxide concentration between both trails (Post-supplementation $30 \mathrm{~min}$ - PLA: $19.3 \pm 4.7 \mu \mathrm{mol} . \mathrm{L}^{-1}$ vs. SP: $18.9 \pm 4.4 \mu \mathrm{mol} . \mathrm{L}^{-1}$ and Post-supplementation $60 \mathrm{~min}$ - PLA: $21.3 \pm 6.5 \mu \mathrm{mol}$. L $^{-1}$ vs. SP: $20.3 \pm 4.9 \mu \mathrm{mol} . \mathrm{L}^{-1}$ ). In addition, no difference was detected for arterial blood pressure. Conclusion: the dietary supplement analyzed in the present study failed to increase nitric oxide endogenous production.
\end{abstract}

Keywords: amino acids, nitric oxide, vasodilatation, blood pressure.

\section{INTRODUÇÃO}

Os mecanismos responsáveis pela hipertrofia muscular, em resposta ao treinamento de força, não estão, totalmente, elucidados ${ }^{(1)}$. Estudos recentes sugerem que esse tipo de exercício promove o acúmulo de substâncias (ex.: lactato) no meio intra e extracelular, que seriam capazes de desencadear a ativação do sistema simpático e a liberação de hormônios hipofisários ${ }^{(2,3)}$. 0 acúmulo desses metabólitos seria responsável por traduzir a sobrecarga utilizada no treinamento em estímulo para a síntese proteica adaptativa ${ }^{(4)}$.

Vale ressaltar que não existem estudos que relacionem o efeito das alterações agudas no perfil hormonal/metabólico em resposta ao treino de força e a adaptação crônica (longo prazo) induzida por esse tipo de treinamento físico ${ }^{(2)}$. Nesse contexto, várias teorias foram elaboradas para explicar como essas respostas agudas estimulariam a hipertrofia do músculo esquelético. Considerando os possíveis mecanismos fisiológicos envolvidos nesse processo, alguns autores propuseram a hipótese da supercirculação. Esta sugere que o incremento do fluxo sanguíneo para o músculo seria um dos estímulos que poderiam potencializar a hipertrofia ${ }^{(5)}$. No caso do treinamento de força, esse aumento na circulação ocorreria, predominantemente, ao término do exercício. Essa resposta resultaria em maior aporte de nutrientes e hormônios para o músculo, bem como a remoção acelerada de subprodutos do metabolismo.

O NO é um vasodilatador endógeno que tem sido investigado, com crescente interesse, devido a seu possível envolvimento nos efeitos cardioprotetores promovidos pelo exercício( ${ }^{(6)}$. Com base na hipótese da supercirculação, suplementos nutricionais, supostamente, precursores/ 
estimuladores da síntese endógena de NO têm sido comercializados com grande sucesso. No entanto, não existem evidências que comprovem que esses suplementos potencializem a hipertrofia por meio da produção de $\mathrm{NO}$ e a subsequente vasodilatação.

É importante destacar que, sendo um radical livre, não é possível disponibilizar a referida substância para o consumo oral. Na realidade, esses produtos possuem aminoácidos que, conforme alegam os fabricantes, seriam capazes de aumentar a produção de NO.

Os poucos estudos que investigaram o efeito desses suplementos nutricionais sobre a produção de $\mathrm{NO}$ e a subsequente vasodilatação, em indivíduos saudáveis durante o repouso, são controversos ${ }^{(7-11)}$. A maioria dos estudos realizados com suplementação ou infusão de aminoácidos, mais especificamente L-arginina, que demonstram efeitos positivos, utilizou indivíduos que apresentavam limitações na fisiologia do endotélio(11,12). Portanto, apesar do grande apelo comercial desses suplementos nutricionais, a comprovação científica da eficiência desses produtos, no sentido de potencializar as respostas agudas e/ou crônicas do treinamento de força, ainda precisa ser estabelecida. Com base nesse hiato, o objetivo do presente estudo foi investigar o efeito da ingestão de um suplemento nutricional à base de proteínas e aminoácidos sobre a concentração plasmática de NO.

\section{MÉTODOS}

\section{Sujeitos}

Foram selecionados 12 sujeitos do sexo masculino, sedentários e saudáveis (idade: $28 \pm 2$ anos; peso: $74 \pm 3,0 \mathrm{~kg}$; altura: $175 \pm 4,0 \mathrm{~cm}$; IMC: $\left.24 \pm 1,0 \mathrm{~kg} / \mathrm{m}^{2}\right)$. O experimento seguiu a resolução específica do Conselho Nacional de Saúde (n 196/96), sendo aprovado pelo Comitê de Ética em Pesquisa do UniFMU (CAAE n 0003.0.254.000-05). Todos os participantes foram informados, detalhadamente, sobre os procedimentos utilizados. Os mesmos concordaram em participar de maneira voluntária do estudo, assinando um termo de consentimento informado e proteção da privacidade.

\section{Protocolo de suplementação}

O produto avaliado foi o Nitro NO2 ${ }^{\circledR}$ Protein Time Release (900g) (Probiótica, Brasil; registro no Ministério da Saúde n 4.9377.0010). Logo após a realização do experimento, o referido produto deixou de ser fabricado, sendo substituído por outro com constituição semelhante. O SP (ou PLA) foi administrado, seguindo o modelo randomizado duplo-cego cruzado. Tanto o SP quanto o PLA foram ministrados na forma de uma bebida adoçada artificialmente, a fim de que não fossem percebidas alterações no paladar. Além disso, as soluções foram ministradas em um recipiente que não permitia visualização da bebida, dificultando a identificação das mesmas. Na primeira semana, os indivíduos se apresentaram para coleta de amostras de sangue 24 horas antes (24) e imediatamente antes (0) do consumo do PLA ou do SP. Além das amostras sanguíneas, a pressão arterial e a frequência cardíaca também foram mensuradas. Os indivíduos foram instruídos a permanecer em jejum por, aproximadamente, 12 horas antes das coletas pré-suplementação. O SP foi consumido conforme as instruções fornecidas pelo fabricante. Os participantes foram divididos entre as condições experimentais (PLA e SP) aleatoriamente. Nessa ocasião, metade da amostra $(n=6)$ consumiu PLA e a outra metade $(n=6)$ ingeriu o SP. Trinta e 60 minutos após a suplementação, amostras de sangue foram, novamente, coletadas. Concomitantemente, a pressão arterial e frequência cardíaca também foram mensuradas. Na semana seguinte, todos os procedimentos foram repetidos, alternando-se a substância a ser consumida. Os indivíduos que, previamente, haviam consumido o PLA, receberam o SP e vice-versa.

\section{Composição do Nitro $\mathrm{NO}^{\circledR}$}

Segundo informações fornecidas pelo fabricante, o Nitro NO2 ${ }^{\circledR}$ Protein Time Release (Probiótica ${ }^{\circledR}$, Brasil; registro no Ministério da Saúde n 4.9377.0010) é constituído de $7 \mathrm{~g}$ de carboidratos, $31 \mathrm{~g}$ de proteínas, $3,5 \mathrm{~g}$ de gorduras totais, $125 \mathrm{mg}$ de cálcio, $0,3 \mathrm{mg}$ de ferro, $100 \mathrm{mg}$ de

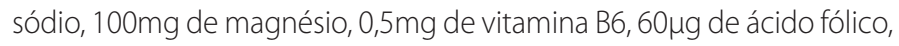
$2.555 \mathrm{mg}$ de L-leucina, $2.473 \mathrm{mg}$ de L-isoleucina e $2.180 \mathrm{mg}$ de L-valina. O conteúdo calórico de uma porção $45 \mathrm{~g}$ do referido suplemento é de 180kcal. É importante destacar que não foi ajuizada a veracidade do conteúdo descrito no rótulo, uma vez que a intenção do estudo foi avaliar a eficiência do produto na forma que o mesmo é comercializado, ficando, portanto, toda a responsabilidade por essas informações por conta do fabricante. Vale notar que a classificação do suplemento como proteico, o que inclusive foi utilizado no título deste artigo, foi fornecida pelo fabricante, e não pelos autores do trabalho.

\section{Protocolo de coleta de sangue}

Para as coletas realizadas antes da suplementação (24 e 0), cada participante compareceu ao laboratório, tendo observado jejum por aproximadamente 12 horas. A coleta de sangue foi realizada no antebraço, utilizando-se tubos a vácuo, nos quais foram extraídos aproximadamente $10 \mathrm{~mL}$ de sangue (Vacutainer ${ }^{\circ}$, EUA). Cada tubo continha 0,5mL de solução com 19,2mg de citrato de sódio e 2,0mg de ácido cítrico. As amostras de sangue foram centrifugadas a $1.500 \mathrm{rpm}$ a $4^{\circ} \mathrm{C}$. O plasma foi extraído e, posteriormente, estocado no freezer a $-80^{\circ} \mathrm{C}$.

\section{Determinação do NO plasmático}

O NO foi avaliado em alíquotas do plasma, previamente desproteinizadas, conforme descrito por Sun et al. ${ }^{(13)}$. O nitrato e o nitrito no plasma foram convertidos em NO. Posteriormente, conforme protocolo de Hampl et al.(14), a concentração de NO foi determinada por quimioluminescência através do analisador NOATM280 (Sievers Inc ${ }^{\odot}$, EUA). Nesse procedimento, a linearidade deve ser menor que $\leq 1 \%$. Logo, o analisador foi calibrado para que a linearidade fosse mantida ao longo de todo o processo de mensuração. A variabilidade entre os ensaios foi menor que 10\%. Os valores estão expressos em $\mu$ mol. L $^{-1}$. Todos os participantes foram instruídos a manter uma dieta restrita em nitrito/nitrato no dia anterior ao do início do experimento. Dessa forma, é possível assegurar que a dosagem de NO não foi afetada pela dieta.

\section{Mensuração da pressão arterial e da frequência cardíaca}

Antes do procedimento de mensuração, (8:00-10:00h), os participantes foram mantidos sentados por cinco minutos. Posteriormente, a pressão arterial foi mensurada de acordo com a American Heart Association, que preconiza a realização de três medidas e a adoção do valor médio das mesmas ${ }^{(15)}$. Os indivíduos não realizaram nenhum tipo de atividade física durante o período (24 horas) que antecedeu o experimento. Os sujeitos também não ingeriram cafeína ou álcool antes do experimento. A frequência cardíaca foi mensurada continuamente por meio de um frequencímetro modelo Polar Vantage NV ${ }^{\odot}$ (Polar ${ }^{\odot}$, Finlândia).

\section{Análise estatística}

Os resultados foram analisados por meio de ANOVA (de dois caminhos, tempo x suplementação). O nível de significância adotado foi de $p<0,05$. A estatística foi realizada por meio do programa SPSS 10.0 for Windows (SPSS ${ }^{\circ}$, EUA). Os resultados estão expressos como média \pm desvio padrão.

\section{RESULTADOS}

A concentração plasmática de $\mathrm{NO}$ não aumentou após o consumo do SP (pré-suplementação SP: 24h - 18,2 \pm 2,5 e imediatamente antes

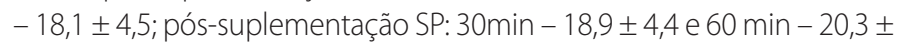
$4,9 \mu \mathrm{mol} . \mathrm{L}^{-1}$ ) (tabela 1). Ao comparar as condições experimentais, nas quais 
foram administrados o PLA e o SP, também não foram detectadas diferenças significativas na concentração plasmática de NO (pré-suplementação PLA: $24 \mathrm{~h}$ 18,8 \pm 2,7 e imediatamente antes - 17,6 2 3,2; pós-suplementação PLA: 30min - 19,3 \pm 4,7 e 60 min - 21,3 $\pm 6,5 \mu$ mol. $^{-1}$ ) (tabela 1).

Tabela 1. Concentração plasmática de NO ( $\mu$ mol..$\left.^{-1}\right)$ pré-suplementação (24 horas e imediatamente antes) e pós-suplementação (30 e 60 minutos) com placebo (PLA) e com suplemento proteico (SP) em indivíduos saudáveis

\begin{tabular}{c|c|c|c|c}
\hline \multirow{2}{*}{ NO $\left(\boldsymbol{\mu m o l} . \mathrm{L}^{-1}\right)$} & \multicolumn{2}{|c|}{ Pré } & \multicolumn{2}{c}{ Pós } \\
\cline { 2 - 5 } & 24 & 0 & 30 & 60 \\
\hline $\mathrm{PLA}(\mathrm{n}=12)$ & $18,8 \pm 2,7$ & $17,6 \pm 3,2$ & $19,3 \pm 4,7$ & $21,3 \pm 6,5$ \\
\hline $\mathrm{SP}(\mathrm{n}=12)$ & $18,2 \pm 2,5$ & $18,1 \pm 4,5$ & $18,9 \pm 4,4$ & $20,3 \pm 4,9$ \\
\hline
\end{tabular}

Valores expressos em média \pm desvio padrão.

A pressão arterial média, a pressão arterial sistólica, a pressão arterial diastólica (tabelas 2, 3 e 4) e a frequência cardíaca (tabela 5) não sofreram alteração em relação aos valores aferidos, previamente, à suplementação proteica. Também não foi evidenciada diferença nesses parâmetros em relação à situação na qual foi consumida a substância PLA.

Tabela 2. Pressão arterial sistólica (PAS) ( $\mathrm{mmHg}$ ) pré-suplementação (24 horas e imediatamente antes) e pós-suplementação (30 e 60 minutos) com placebo (PLA) e com suplemento proteico (SP) em indivíduos saudáveis

\begin{tabular}{c|c|c|c|c}
\hline \multirow{2}{*}{ PAS (mmHg) } & \multicolumn{2}{|c|}{ Pré } & \multicolumn{2}{c}{ Pós } \\
\cline { 2 - 5 } & 24 & 0 & 30 & 60 \\
\hline PLA $(n=12)$ & $113,5 \pm 6,4$ & $114,5 \pm 4,8$ & $116,0 \pm 4,7$ & $113,2 \pm 6,2$ \\
\hline SP $(n=12)$ & $114,0 \pm 5,7$ & $114,7 \pm 4,3$ & $115,5 \pm 4,6$ & $111,7 \pm 5,6$ \\
\hline
\end{tabular}

Valores expressos em média \pm desvio padrão.

Tabela 3. Pressão arterial diastólica (PAD) $(\mathrm{mmHg})$ pré-suplementação (24 horas e imediatamente antes) e pós-suplementação (30 e 60 minutos) com placebo (PLA) e com suplemento proteico (SP) em indivíduos saudáveis

\begin{tabular}{c|c|c|c|c}
\hline \multirow{2}{*}{$\begin{array}{c}\text { PAD } \\
(\mathbf{m m H g})\end{array}$} & \multicolumn{2}{|c|}{ Pré } & \multicolumn{2}{c}{ Pós } \\
\cline { 2 - 5 } & 24 & 0 & 30 & 60 \\
\hline $\mathrm{PLA}(\mathrm{n}=12)$ & $79,8 \pm 0,6$ & $79,5 \pm 0,9$ & $79,7 \pm 0,8$ & $80,2 \pm 6,1$ \\
\hline $\mathrm{SP}(\mathrm{n}=12)$ & $80,7 \pm 1,2$ & $80,0 \pm 1,0$ & $79,8 \pm 0,6$ & $80,2 \pm 3,6$ \\
\hline
\end{tabular}

Valores expressos em média \pm desvio padrão.

Tabela 4. Pressão arterial média (PAM) (mmHg) pré-suplementação (24 horas e imediatamente antes) e pós-suplementação (30 e 60 minutos) com placebo (PLA) e com suplemento proteico (SP) em indivíduos saudáveis

\begin{tabular}{c|c|c|c|c}
\hline \multirow{2}{*}{$\begin{array}{c}\text { PAM } \\
(\mathbf{m m H g})\end{array}$} & \multicolumn{2}{|c|}{ Pré } & \multicolumn{2}{c}{ Pós } \\
\cline { 2 - 5 } & 24 & 0 & 30 & 60 \\
\hline PLA $(\mathrm{n}=12)$ & $91,1 \pm 2,2$ & $91,2 \pm 1,8$ & $91,8 \pm 1,7$ & $91,2 \pm 4,2$ \\
\hline $\mathrm{SP}(\mathrm{n}=12)$ & $91,8 \pm 2,0$ & $91,6 \pm 1,8$ & $91,7 \pm 1,9$ & $90,7 \pm 3,6$ \\
\hline
\end{tabular}

Valores expressos em média \pm desvio padrão.

Tabela 5. Frequência cardíaca (bpm) pré-suplementação (24 horas e imediatamente antes) e pós-suplementação (30 e 60 minutos) com placebo (PLA) e com suplemento proteico (SP) em indivíduos saudáveis

\begin{tabular}{c|c|c|c|c}
\hline \multirow{2}{*}{ FC (bpm) } & \multicolumn{2}{|c|}{ Pré } & \multicolumn{2}{c}{ Pós } \\
\cline { 2 - 5 } & 24 & 0 & 30 & 60 \\
\hline PLA $(n=12)$ & $71,5 \pm 6,2$ & $70,3 \pm 4,9$ & $69,9 \pm 3,9$ & $69,0 \pm 3,7$ \\
\hline SP $(n=12)$ & $72,1 \pm 5,8$ & $70,5 \pm 4,6$ & $71,0 \pm 1,7$ & $68,2 \pm 1,5$ \\
\hline
\end{tabular}

Valores expressos em média \pm desvio padrão.

\section{DISCUSSÃO}

O principal achado do presente trabalho foi que o suplemento avaliado não promoveu alteração na concentração plasmática de NO, em indivíduos adultos saudáveis, na situação de repouso. O comportamento da pressão arterial (média, sistólica e diastólica), parâmetro, potencialmente, afetado pela resposta de vasodilatação, também não foi alterado. Obviamente, os métodos utilizados na presente investigação não permitem descartar, totalmente, o fato de que a vasodilatação tenha sido contrabalançada por algum mecanismo fisiológico (ex:: ação do sistema simpático).

O NO é um potente vasodilatador que tem despertado o interesse dos pesquisadores que investigam os efeitos cardioprotetores do exercício ${ }^{16}$. É importante ressaltar que os estudos que demonstraram aumento na concentração de NO, em função da utilização de precursores, tanto em animais quanto em seres humanos, foram conduzidos em condições patológicas, como doenças cardiovasculares ou fatores de risco para as mesmas ${ }^{(11,12)}$. Entretanto, esses resultados, na maioria das vezes, não são reproduzidos em indivíduos saudáveis sem comprometimento endotelia|(12).

Portanto, apesar da popularidade mundial de suplementos "precursores" de NO, a coletânea de dados científicos disponíveis na literatura não permite definir as reais implicações desse tipo de suplementação dietética sobre as respostas crônicas do treinamento físico. Outro fato a ser destacado é que esses poucos resultados obtidos se referem ao exercício de endurance ${ }^{(16)}$, e não ao exercício de força, como modelo para verificação do efeito do NO sobre a vasodilatação.

Atualmente, não existem trabalhos publicados comprovando a importância da vasodilatação para o ganho de força ou hipertrofia decorrentes do treinamento de força. Tipton et al.(17), por exemplo, demonstraram apenas que o aumento da síntese proteica após o exercício poderia estar relacionado com o maior do fluxo sanguíneo para a musculatura exercitada, após a sessão de treino. Porém, neste estudo não foi feita nenhuma avaliação ou inferência da participação da síntese endógena NO na resposta de vasodilatação.

Recentemente, um trabalho de nosso grupo demonstrou significativa resposta de hipertrofia do músculo esquelético, após a utilização de um modelo experimental de sobrecarga mecânica em ratos ${ }^{(18)}$. Nesse trabalho, foi mensurada a expressão de duas isoformas (neuronal e induzida) da óxido nítrico sintase (NOS) no músculo esquelético. Ambas as isoformas apresentaram aumento de expressão da NOS após o modelo de sobrecarga mecânica ${ }^{(18)}$. O NO também é um potente estimulador das células-satélite ${ }^{(19,20)}$. Consequentemente, além da hipótese da supercirculação, outro provável efeito da estimulação da síntese de NO sobre o processo de hipertrofia poderia ser modulado pela ativação das células-satélite.

Em função da total ausência de dados a respeito, o objetivo deste trabalho foi investigar o efeito de um suplemento nutricional comercializado com intuito de estimular a síntese endógena de NO. Ainda que alguns estudos demonstrem que produção endógena de NO pode ser estimulada por suplementos nutricionais, existe a necessidade fundamental de provar que a referida substância vasoativa está, de alguma forma, envolvida com as adaptações crônicas ao treinamento de força em indivíduos saudáveis.

Infelizmente, não existem na literatura dados que possam ser comparados com os achados da presente investigação. A ausência de outros estudos sobre o uso de suplementos nutricionais, à base de proteínas ou aminoácidos de cadeia ramificada (BCAA), a fim de estimular a produção de NO, é justificada pelo fato de que o precursor do NO é a L-arginina, e não os BCAA ${ }^{(11,21)}$.

A arginina (ácido 2-amino-5-guanidino-pentanoico) é um aminoácido condicionalmente essencial(22), envolvido em vários processos metabólicos, como a síntese de creatina e ornitina. As principais fontes 
de arginina são constituídas pela dieta, pela síntese endógena e pelo turnover de outras proteínas. Cerca de 40\% do pool de arginina proveniente da dieta é degradado no próprio intentino, antes de alcançar a circulação ${ }^{(23)}$. No jejum, a maior parte (85\%) da arginina circulante é proveniente do turnover proteico e o restante é originada da síntese endógena que ocorre no intestino e no rim ${ }^{(23)}$.

A administração de arginina visa aumentar a produção de NO. Existem diversas técnicas utilizadas para aumentar a disponibilidade desse aminoácido, destacando-se a intra-arterial, a intravenosa e a suplementação oral. A concentração plasmática de arginina é de aproximadamente $100 \mu \mathrm{mol} \cdot \mathrm{L}^{-1}$. Passados 30 minutos, após da infusão de $30 \mathrm{~g}$ de arginina, foi observado que sua concentração no plasma aumentou para aproximadamente $7.000 \mu \mathrm{mol} . \mathrm{L}^{-1(24)}$. Diversos estudos avaliaram o efeito da infusão de arginina em pacientes apresentando doenças cardiovasculares(25), hipercolesteronemia $^{(26)}$, hipertensão(27) ${ }^{(2)}$ diabetes ${ }^{(28)}$ e comprometimentos vasculares periféricos ${ }^{(29)}$, e, em todas as circunstâncias, a infusão de arginina aumentou a produção/função do NO.

Outra estratégia de administração, com menor eficiência, é realizada por via oral. Devido ao fato do significativo catabolismo realizado no enterócito, a ingestão de arginina pode não ser a melhor opção. A biodisponibilidade da arginina suplementada por via oral varia de 21 a $67 \%(7,11,23,30)$. Atualmente, diversos suplementos nutricionais à base de arginina são comercializados com intuito de melhorar a função endotelial. Entretanto, conforme já enfatizado, poucos estudos avaliaram a eficiência deses produtos em associação com o treinamento físico. Mais especificamente, com relação à suplementação de arginina em associação com o treinamento de força, não existem estudos até o presente momento.
Vale lembrar que as pesquisas que observaram o efeito positivo de preservar a massa magra em decorrência da suplementação com arginina foram realizadas com indivíduos portadores de queimaduras e com sepse ${ }^{(12)}$. Logo, é muito difícil extrapolar esses achados para indivíduos fisicamente ativos que buscam hipertrofia.

Mesmo assim, tais efeitos, observados em condições extremas, dependem de quanto a síntese endógena ou a dieta estão, ou não, fornecendo arginina em quantidade suficiente ${ }^{(12)}$. Outro aspecto a ser considerado é a maneira pela qual os aminoácidos são disponibilizados. Existem resultados bastante conflitantes entre suplementação oral e infusão venosa ${ }^{(7,9,10)}$.

Apesar de alguns estudos comprovarem o efeito estimulador da administração de arginina sobre a síntese endógena de NO, é importante mencionar que, no produto avaliado, o referido aminoácido não constava na descrição dos ingredientes presentes no rótulo. Esse fato merece destaque, uma vez que o produto testado foi comercializado como estimulador da síntese de NO.

\section{CONCLUSÃO}

O suplemento proteico avaliado não promoveu aumento na concentração plasmática de NO. O referido suplemento também não alterou o comportamento da pressão arterial de indivíduos adultos saudáveis em repouso.

Todos os autores declararam não haver qualquer potencial conflito de interesses referente a este artigo.

\section{REFERÊNCIAS BIBLIOGRÁFICAS}

1. Goldspink G, Harridge S. Cellular and molecular aspects of adaptation in skeletal muscle. In: Komi PV, editor. Strength and power in sport. Oxford: Blackwell Science, 2003, 231-51.

2. Goto K, Ishii N, Kizuka T, Takamatsu K. The impact of metabolic stress on hormonal responses and muscular adaptations. Med Sci Sports Exerc. 2005;37(6):955-63.

3. Kawada S, Ishii N. Skeletal muscle hypertrophy after chronic restriction of venous blood flow in rats. Med Sci Sports Exerc. 2005;37(7):1144-50.

4. Viru A, Viru M. Metabolic adaptation in training. In: Viru A, Viru M, editors. Biochemical monitoring of sport training. Champain: Human Kinetics, 2001, 11-25.

5. Zatsiorsky VM, editor. Ciência e prática do treinamento de força. São Paulo: Phorte Editora, 1999.

6. Tatchum-Talon R, Schulz R, McNeill JR, Khadour FH. Upregulation of neuronal nitric oxide synthase in skeletal muscle by swin training. Am J Physiol Heart Circ Physiol. 2000;279(4):H1757-66.

7. Bode-Boger SM, Boger RH, Galland A, Tsikas D, Frolich JC. L-arginine-induced vasodilation in healthy humans: pharmacokinetic-pharmacodynamic relationship. Br J Clin Pharmacol. 1998;46(5):489-97.

8. Siani A, Pagano E, lacone R, lacoviello L, Scopacasa F, Strazzullo P. Blood pressure and metabolic changes during dietary L-arginine supplementation in humans. Am J Hypertens. 2000;13(5 Pt 1):547-51.

9. Bode-Boger SM, Muke J, Surdacki A, Brabant G, Boger RH, Frolich JC. Oral L-arginine improves endothelial function in healthy individuals older than 70 years. Vasc Med. 2003;8(2):77-81.

10. Evans RW, Fernstrom JD, Thompson J, Morris SM Jr, Kuller LH. Biochemical responses of healthy subjects during dietary supplementation with L-arginine. J Nutr Biochem. 2004;15(9):534-9.

11. Huynh NN, Chin-Dusting J. Amino acids, arginase and nitric oxide in vascular health. Clin Exp Pharmacol Physiol. 2006;33(1-2):1-8.

12. Paddon-Jones D, Borshein E, Wolfe RR. Potential ergogenic effects of arginine and creatine supplementation. J Nutr. 2004;134(10 Spl):2888S-2894S; discussion 295S.

13. Sun J, Zhang X, Broderick M, Fein H. Measurement of nitric oxide production in biological system by using griess reaction assay. Sensor. 2003;3:276-84.

14. Hampl V, Tristani-Firouzi M, Nelson DP, Archer SL. Chronic infusion of nitric oxide in experimental pulmonary hypertension: pulmonary pressure-flow analysis. Eur Respir J. 1996;9(7):1475-81.

15. Fletcher GF, Froelicher VF, Hartley LH, Haskell WL, Pollock ML. Exercise standards. A statement for health professionals from the American Heart Association. Circulation. 1990;82(6):2286-322.

16. Green DJ, Maiorana A, O'Driscoll G, Taylor R. Effect of exercise training on endothelium-derived nitric oxide function in humans. J Physiol. 2004;561(Pt 1):1-25.
17. Tipton KD, Rasmussen BB, Miller SL, Wolf SE, Owens-Stovall SK, Petrini BE, et al. Timing of amino acid-carbohydrate ingestion alters anabolic response of muscle to resistance exercise. Am J Physiol Endocrinol Metab. 2001;281(2):E197-206.

18. Aoki MS, Miyabara EH, Soares AG, Saito ET, Moriscot AS. mTOR pathway inhibition attenuates skeletal muscle growth induced by stretching. Cell Tissue Res. 2006;324(1):149-56.

19. Anderson JE, Wozniak AC. Satellite cell activation on fibers: modeling events in vivo - an invited review. Can J Physiol Pharmacol. 2004;82(5):300-10.

20. Tatsumi R, Liu X, Pulido A, Morales M, Sakata T, Dial S, et al. Satellite cell activation in stretched skeletal muscle and the role of nitric oxide and hepatocyte growth factor. Am J Physiol Cell Physiol. 2006;290(6):C1487-94.

21. Stamler JS, Meissner G. Physiology of nitric oxide in skeletal muscle. Physiol Rev. 2001;81(1):209-37. Review.

22. Rose W. The nutritive significance of the amino acids and certain related compounds. Science. 1937;86(2231):298-300.

23. Wu G, Morris SM Jr. Arginine metabolism: nitric oxide and beyond. Biochem J. 1998;336(Pt 1):1-17.

24. Rhodes $P$, Barr CS, Struthers AD. Arginine, lysine and ornithine as vasodilators in the forearm of man. Eur J Clin Invest. 1996;26(4):325-31.

25. Tousoulis D, Davies G, Tentolouris C, Crake T, Toutouzas P. Coronary stenosis dilatation induced by L-arginine. Lancet. 1997;349(9068):1812-3.

26. Creager MA, Gallagher SJ, Girerd XJ, Coleman SM, Dzau VJ, Cooke JP. L-arginine improves endotheliumdependent vasodilation in hypercholesterolemic humans. J Clin Invest. 1992;90(4):1248-53.

27. Schlaich MP, Ahlers BA, Parnell MM, Kaye DM. beta-Adrenoceptor-mediated, nitric-oxide-dependent vasodilatation is abnormal in early hypertension: restoration by L-arginine. J Hypertens. 2004;22(10):1917-25.

28. Pieper GM, Siebeneich W, Dondlinger LA. Short-term oral administration of L-arginine reverses defective endothelium-dependent relaxation and cGMP generation in diabetes. Eur J Pharmacol. 1996;317(2-3):317-20.

29. Boger RH, Bode-Boger SM, Thiele W, Creutzig A, Alexander K, Frolich JC. Restoring vascular nitric oxide formation by L-arginine improves the symptoms of intermittent claudication in patients with peripheral arterial occlusive disease. J Am Coll Cardiol. 1998;32(5):1336-44.

30. Tangphao O, Chalon S, Moreno Jr H, Hoffman BB, Blaschke TF. Pharmacokinetics of L-arginine during chronic administration to patients with hypercholesterolaemia. Clin Sci (Lond). 1999;96(2):199-207. 